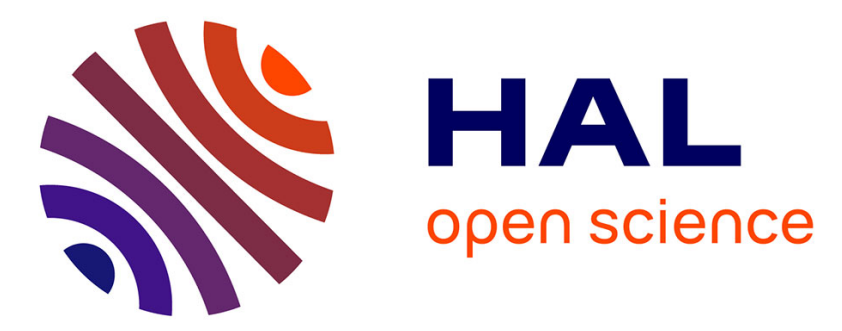

\title{
Stability Analysis of Systems with Time-varying Delays via the Second-order Bessel-Legendre Inequality
}

\author{
Kun Liu, Alexandre Seuret, Yuanqing Xia
}

\section{To cite this version:}

Kun Liu, Alexandre Seuret, Yuanqing Xia. Stability Analysis of Systems with Time-varying Delays via the Second-order Bessel-Legendre Inequality. Automatica, 2017, 76 (2), pp.138-142. 10.1016/j.automatica.2016.11.001 . hal-01382473

\section{HAL Id: hal-01382473 \\ https://hal.science/hal-01382473}

Submitted on 17 Oct 2016

HAL is a multi-disciplinary open access archive for the deposit and dissemination of scientific research documents, whether they are published or not. The documents may come from teaching and research institutions in France or abroad, or from public or private research centers.
L'archive ouverte pluridisciplinaire HAL, est destinée au dépôt et à la diffusion de documents scientifiques de niveau recherche, publiés ou non, émanant des établissements d'enseignement et de recherche français ou étrangers, des laboratoires publics ou privés. 


\title{
Stability Analysis of Systems with Time-varying Delays via the Second-order Bessel-Legendre Inequality *
}

\author{
Kun Liu ${ }^{a}$ Alexandre Seuret ${ }^{\mathrm{b}, \mathrm{c}}$ Yuanqing Xia ${ }^{\mathrm{a}}$ \\ ${ }^{a}$ School of Automation, Beijing Institute of Technology, 100081 Beijing, China. \\ ${ }^{\mathrm{b}}$ CNRS, LAAS, 7 avenue du Colonel Roche, 31077 Toulouse, France. \\ ${ }^{\mathrm{c}}$ Univ de Toulouse, LAAS, F-31400 Toulouse, France.
}

\begin{abstract}
This paper is concerned with the delay-dependent stability of systems with time-varying delays. The novelty relies on the use of the second-order Bessel-Legendre integral inequality which is less conservative than the Jensen and Wirtinger-based inequalities. Unlike similar contributions, the features of this inequality are fully integrated into the construction of augmented Lyapunov-Krasovskii functionals leading to novel stability criteria expressed in terms of linear matrix inequalities. The stability condition is tested on some classical numerical examples illustrating the efficiency of the proposed method.
\end{abstract}

Key words: Time-varying delays, integral inequality, Lyapunov method.

\section{Introduction}

Over the last two decades, the delay-dependent stability analysis of systems with time-varying delays via Lyapunov-Krasovskii functional (LKF) method has received much attention (see e.g., [1], [10] and the references therein). The crucial technical steps regarding this method are related to both the selection of the functional and the use of accurate bounding methods to derive linear matrix inequalities (LMIs).

Among the bounding methods, the Jensen inequality [2] has been widely adopted, although at the price of an unavoidable conservatism. Recently, much attention has been paid to reducing this conservatism. A recent direction of research consists in deriving extended-like Jensen inequalities, which encompasses Jensen inequality through the introduction of additional quadratic terms. The first result in this direction led to the socalled Wirtinger-based inequality developed in $[6,9]$.

\footnotetext{
* This work was supported by the National Natural Science Foundation of China (grant no. 61503026, 71601019), the ANR project SCIDiS contract number 15-CE23-0014, and the Foundation of Beijing Institute of Technology (grant no. 20150642003). The material in this paper was not presented at any conference.

E-mail addresses: kunliubit@bit.edu.cn (K. Liu), aseuret@laas.fr (A. Seuret), xia_yuanqing@bit.edu.cn (Y. Xia)
}

Then, further extensions were derived by Zeng et al. using a free-matrix-based integral inequality [11]. More recently, generalized integral inequalities were developed in $[7,8]$ based on Bessel inequality and Legendre polynomials, which includes Jensen and Wirtingerbased inequalities and also the recent inequalities based on auxiliary functions-based inequality $([5],[12])$ as particular cases. The main interest of such inequalities is that the conservatism can be reduced arbitrarily. These new inequalities have been mainly employed to the case of constant discrete or distributed delays (see [8],[12]). The first attempt to derive stability condition for timevarying discrete delay was proposed in [5]. In this paper, we pursue the method provided in [5] and derive more accurate conditions.

In this paper, we develop novel stability criteria for linear systems with time-varying delays using the particular case of the second-order Bessel-Legendre inequality (i.e. the Bessel-Legendre inequality from [8] with $N=2$, where $N$ is the degree of Legendre polynomials) of the integral inequalities proposed in $[7,8]$, that recovers the inequality provided in $[5,12]$. The main contributions are as follows:

1. The features of the limited Bessel-Legendre inequality are fully integrated into the construction of the LKFs.

2. Less conservative stability criteria are derived in terms of LMIs although the computational complex- 
ity is higher.

Numerical examples taken from the literature illustrate the efficiency of our results. In particular, the numerical results for the constant delay case coincide with the ones achieved in [12] and are very close to the analytical bounds of constant delays preserving the stability.

Throughout the paper, in addition to usual notations, the set $\mathbb{S}_{+}^{n}$ denotes the set of symmetric positive definite matrices. For any matrices $A, B, \operatorname{diag}(A, B)$ stands for the matrix $\left[\begin{array}{cc}A & 0 \\ 0 & B\end{array}\right]$. Moreover, for any square matrix $A$, we define $\operatorname{He}(A)=A+A^{T}$.

\section{Problem formulations}

\subsection{System data}

Consider a linear system with time-varying delays:

$$
\left\{\begin{array}{l}
\dot{x}(t)=A x(t)+A_{1} x(t-h(t)), \quad t \geq 0 \\
x(t)=\phi(t), \quad-h_{2} \leq t \leq 0
\end{array}\right.
$$

where $x(t) \in \mathbb{R}^{n}$ is the state vector, $A, A_{1} \in \mathbb{R}^{n \times n}$ are constant matrices, and $\phi$ is the initial condition. The time-varying delay $h(t)$ is continuous and satisfies

$$
0 \leq h_{1} \leq h(t) \leq h_{2}, \quad h_{12} \triangleq h_{2}-h_{1}
$$

There is no restriction on the derivative of the delay function.

\subsection{Limited Bessel-Legendre inequality}

Let us first recall the inequality that will be the core of the paper. It corresponds to the inequality recently shown in [12], which is also a particular case of the BesselLegendre inequality of [8]. The proof of this inequality can be found in [12] or in [8].

Lemma 1 For a given matrix $R \in \mathbb{S}_{+}^{n}$, any differentiable function $x$ in $[a, b] \rightarrow \mathbb{R}^{n}$, the inequality

$$
\int_{a}^{b} \dot{x}^{T}(u) R \dot{x}(u) \mathrm{d} u \geq \frac{1}{b-a} \Omega^{T} \operatorname{diag}(R, 3 R, 5 R) \Omega
$$

holds, where

$$
\begin{aligned}
& \Omega=\left[\begin{array}{l}
x(b)-x(a) \\
x(b)+x(a)-\frac{2}{b-a} \int_{a}^{b} x(u) \mathrm{d} u \\
x(b)-x(a)-\frac{6}{b-a} \int_{a}^{b} \delta_{a, b}(u) x(u) \mathrm{d} u
\end{array}\right] \\
& \delta_{a, b}(u)=2\left(\frac{u-a}{b-a}\right)-1 .
\end{aligned}
$$

Remark 1 The inequality (3) encompasses the Wirtinger-based inequality of [6] with the help of the third component of the vector $\Omega$. This improvement requires the introduction of an extra signal $\int_{a}^{b} \delta_{a, b}(u) x(u) \mathrm{d} u$ in addition to $\int_{a}^{b} x(u) \mathrm{d} u, x(b)$ and $x(a)$.

\subsection{Parameter-dependent matrix inequalities}

The following lemma is for an alternative formulation of the reciprocally convex combination inequality from [4].

Lemma 2 For any given matrix $R \in \mathbb{S}_{+}^{n}$, assume that there exists a matrix $X \in \mathbb{R}^{n \times n}$ such that $\left[\begin{array}{cc}R & X \\ X^{T} & R\end{array}\right] \succeq 0$. Then, the following inequality holds

$$
\left[\begin{array}{cc}
\frac{1}{\alpha} R & 0 \\
0 & \frac{1}{1-\alpha} R
\end{array}\right] \succeq\left[\begin{array}{cc}
R & X \\
X^{T} & R
\end{array}\right], \quad \forall \alpha \in(0,1)
$$

Alternatively, we present another lemma, which refers to the classical bounding technique [3]

Lemma 3 For any matrices $R_{1} \in \mathbb{S}_{+}^{n}, R_{2} \in \mathbb{S}_{+}^{n}, Y_{1} \in$ $\mathbb{R}^{2 n \times n}$ and $Y_{2} \in \mathbb{R}^{2 n \times n}$, the following inequality holds

$$
\left[\begin{array}{cc}
\frac{1}{\alpha} R_{1} & 0 \\
0 & \frac{1}{1-\alpha} R_{2}
\end{array}\right] \succeq \Theta_{M}(\alpha), \quad \forall \alpha \in(0,1)
$$

where

$$
\begin{aligned}
\Theta_{M}(\alpha)= & \operatorname{He}\left(Y_{1}\left[\begin{array}{ll}
I_{n} & 0_{n \times n}
\end{array}\right]+Y_{2}\left[\begin{array}{ll}
0_{n \times n} & I_{n}
\end{array}\right]\right) \\
& -\alpha Y_{1} R_{1}^{-1} Y_{1}^{T}-(1-\alpha) Y_{2} R_{2}^{-1} Y_{2}^{T} .
\end{aligned}
$$

The notable difference between Lemmas 2 and 3 is that, in Lemma 3, the lower bound depends explicitly on the uncertain parameter $\alpha$. This dependence on $\alpha$ eventually leads to a reduction of conservatism at the price of additional decision variables.

\section{Stability analysis of time-varying delay sys- tems}

In this section, based on Lemma 1 together with Lemma 2 or 3 , two novel stability criteria are provided for system (1) with time-varying delays. For the simplicity of presentation, we will use in this section the 
following notations:

$$
\begin{aligned}
e_{i} & =\left[\begin{array}{lll}
0_{n \times(i-1) n} & I_{n} & 0_{n \times(14-i) n}
\end{array}\right], i=1, \ldots, 14, \\
G_{2} & =\left[\begin{array}{lll}
e_{1}^{T}-e_{2}^{T} & e_{1}^{T}+e_{2}^{T}-2 e_{5}^{T} & e_{1}^{T}-e_{2}^{T}-6 e_{6}^{T}
\end{array}\right]^{T}, \\
G_{3} & =\left[\begin{array}{lll}
e_{2}^{T}-e_{3}^{T} & e_{2}^{T}+e_{3}^{T}-2 e_{7}^{T} & e_{2}^{T}-e_{3}^{T}-6 e_{8}^{T}
\end{array}\right]^{T}, \\
G_{4} & =\left[\begin{array}{lll}
e_{3}^{T}-e_{4}^{T} & e_{3}^{T}+e_{4}^{T}-2 e_{9}^{T} & e_{3}^{T}-e_{4}^{T}-6 e_{10}^{T}
\end{array}\right]^{T}, \\
\Gamma & =\left[\begin{array}{ll}
G_{3}^{T} & G_{4}^{T}
\end{array}\right]^{T}, \quad \Sigma=A e_{1}+A_{1} e_{3} .
\end{aligned}
$$

and

$\eta_{0}(t)=\left[\begin{array}{llll}x^{T}(t) & x^{T}\left(t-h_{1}\right) & x^{T}(t-h(t)) & x^{T}\left(t-h_{2}\right)\end{array}\right]^{T}$,

$\eta_{1}(t)=\frac{1}{h_{1}}\left[\int_{-h_{1}}^{0} x_{t}^{T}(s) \mathrm{d} s \int_{-h_{1}}^{0} \delta_{1}(s) x_{t}^{T}(s) \mathrm{d} s\right]^{T}$,

$\eta_{2}(t)=\frac{1}{h(t)-h_{1}}\left[\int_{-h(t)}^{-h_{1}} x_{t}^{T}(s) \mathrm{d} s \quad \int_{-h(t)}^{-h_{1}} \delta_{2}(s) x_{t}^{T}(s) \mathrm{d} s\right]^{T}$,

$\eta_{3}(t)=\frac{1}{h_{2}-h(t)}\left[\int_{-h_{2}}^{-h(t)} x_{t}^{T}(s) \mathrm{d} s \int_{-h_{2}}^{-h(t)} \delta_{3}(s) x_{t}^{T}(s) \mathrm{d} s\right]^{T}$,

$\eta_{4}(t)=\left(h(t)-h_{1}\right) \eta_{2}(t), \quad \eta_{5}(t)=\left(h_{2}-h(t)\right) \eta_{3}(t)$.

$\eta_{6}(t)=\left[\int_{-h_{2}}^{-h_{1}} x_{t}^{T}(s) \mathrm{d} s \quad h_{12} \int_{-h_{2}}^{-h_{1}} \delta_{4}(s) x_{t}^{T}(s) \mathrm{d} s\right]^{T}$,

and where the functions $\delta_{i}$, for $i=1, \ldots, 4$, which refer to the functions $\delta_{a, b}$ given in Lemma 1 , are given by

$$
\begin{aligned}
\delta_{1}(s) & =2 \frac{s+h_{1}}{h_{1}}-1, & \delta_{2}(s) & =2 \frac{s+h(t)}{h(t)-h_{1}}-1, \\
\delta_{3}(s) & =2 \frac{s+h_{2}}{h_{2}-h(t)}-1, & \delta_{4}(s) & =2 \frac{s+h_{2}}{h_{12}}-1 .
\end{aligned}
$$

\section{1 "Reciprocally convex"-based result}

The following theorem on the asymptotic stability analysis of system (1) is derived from Lemmas 1 and 2 .

Theorem 1 If there exist matrices $P \in \mathbb{S}_{+}^{5 n}, S_{1}, S_{2}$, $R_{1}, R_{2} \in \mathbb{S}_{+}^{n}, N_{1}, N_{2} \in \mathbb{R}^{14 n \times 2 n}$, and a matrix $X \in$ $\mathbb{R}^{3 n \times 3 n}$ such that the LMIs

$$
\Psi=\left[\begin{array}{cc}
\tilde{R}_{2} & X \\
X^{T} & \tilde{R}_{2}
\end{array}\right] \succeq 0, \Phi\left(h_{i}\right)=\Phi_{0}\left(h_{i}\right)-\Gamma^{T} \Psi \Gamma \prec 0,
$$

hold, for $i=1,2$, where, for any $\theta$ in $\mathbb{R}$,

$$
\begin{aligned}
& \Phi_{0}(\theta)=\operatorname{He}\left(G_{1}^{T}(\theta) P G_{0}+N_{1} g_{1}(\theta)+N_{2} g_{2}(\theta)\right)+\hat{S} \\
& +\Sigma^{T}\left(h_{1}^{2} R_{1}+h_{12}^{2} R_{2}\right) \Sigma-G_{2}^{T} \tilde{R}_{1} G_{2}, \\
& \hat{S}=\operatorname{diag}\left(S_{1},-S_{1}+S_{2}, 0_{n \times n},-S_{2}, 0_{10 n \times 10 n}\right), \\
& \tilde{R}_{i}=\operatorname{diag}\left(R_{i}, 3 R_{i}, 5 R_{i}\right), \quad i=1,2, \\
& G_{0}=\left[\begin{array}{lllll}
\Sigma^{T} & e_{1}^{T}-e_{2}^{T} & e_{1}^{T}+e_{2}^{T}-2 e_{5}^{T} & e_{2}^{T}-e_{4}^{T} & \hat{G}_{0}^{T}
\end{array}\right]^{T}, \\
& \hat{G}_{0}=h_{12}\left(e_{2}+e_{4}\right)-2\left(e_{11}+e_{13}\right) \text {, } \\
& G_{1}(\theta)=\left[\begin{array}{lllll}
e_{1}^{T} & h_{1} e_{5}^{T} & h_{1} e_{6}^{T} & e_{11}^{T}+e_{13}^{T} & \hat{G}_{1}^{T}(\theta)
\end{array}\right]^{T}, \\
& \hat{G}_{1}(\theta)=\left(h_{2}-\theta\right)\left(e_{11}+e_{14}\right)+\left(\theta-h_{1}\right)\left(e_{12}-e_{13}\right) \text {, }
\end{aligned}
$$

and

$g_{1}(\theta)=\left(\theta-h_{1}\right)\left[\begin{array}{l}e_{7} \\ e_{8}\end{array}\right]-\left[\begin{array}{l}e_{11} \\ e_{12}\end{array}\right], g_{2}(\theta)=\left(h_{2}-\theta\right)\left[\begin{array}{c}e_{9} \\ e_{10}\end{array}\right]-\left[\begin{array}{l}e_{13} \\ e_{14}\end{array}\right]$.

Then, system (1) is asymptotically stable for all timevarying delays $h(t)$ satisfying $(2)$.

Proof: Consider a Lyapunov functional given by

$$
\begin{aligned}
& \quad \begin{array}{l}
V\left(x_{t}, \dot{x}_{t}\right)=V_{1}\left(x_{t}\right)+V_{2}\left(x_{t}\right)+V_{3}\left(x_{t}, \dot{x}_{t}\right) \\
V_{1}\left(x_{t}\right)=\tilde{x}^{T}(t) P \tilde{x}(t) \\
V_{2}\left(x_{t}\right)=\int_{t-h_{1}}^{t} x^{T}(s) S_{1} x(s) \mathrm{d} s+\int_{t-h_{2}}^{t-h_{1}} x^{T}(s) S_{2} x(s) \mathrm{d} s \\
V_{3}\left(x_{t}, \dot{x}_{t}\right)=h_{1} \int_{-h_{1}}^{0} \int_{t+\theta}^{t} \dot{x}^{T}(s) R_{1} \dot{x}(s) \mathrm{d} s \mathrm{~d} \theta \\
\quad+h_{12} \int_{-h_{2}}^{-h_{1}} \int_{t+\theta}^{t} \dot{x}^{T}(s) R_{2} \dot{x}(s) \mathrm{d} s \mathrm{~d} \theta
\end{array}
\end{aligned}
$$

where

$$
\tilde{x}(t)=\operatorname{col}\left\{x(t), h_{1} \eta_{1}(t), \eta_{6}(t)\right\},
$$

Remark 2 While functionals $V_{2}$ and $V_{3}$ have been already considered in e.g., [4], [9], a first selection for $V_{1}$ is a quadratic term only depending on the instantaneous state vector $x(t)$ (see e.g. [4] among many others). It was shown in [9], that $V_{1}$ needs to be augmented in order to fully benefit from the Wirtinger-based inequality. The extension consists of including $\int_{-h_{1}}^{0} x_{t}(s) \mathrm{d} s$ and $\int_{-h_{2}}^{-h_{1}} x_{t}(s) \mathrm{d} s$, which appear naturally in the Wirtingerbased inequality. Since we are now considering an extented version of the Wirtinger-based inequality (i.e. Bessel-Legendre), the term $V_{1}$ in (9) also needs to include the two additional signals $\int_{-h_{1}}^{0} \delta_{1}(s) x_{t}(s) \mathrm{d} s$ and $\int_{-h_{2}}^{-h_{1}} \delta_{4}(s) x_{t}(s) \mathrm{d}$ s issued from (3). This state augmentation in $V_{1}$ was not considered yet in the literature and we will demonstrate, on examples, its necessity.

The objective of the next developments consists in finding an upper bound of the derivative of $V\left(x_{t}, \dot{x}_{t}\right)$ along 
the trajectories of (1) using the augmented vector

$$
\zeta(t)=\operatorname{col}\left\{\eta_{0}(t), \eta_{1}(t), \eta_{2}(t), \eta_{3}(t), \eta_{4}(t), \eta_{5}(t)\right\}
$$

where $\eta_{i}(t)$, for $i=0, \ldots, 5$, are given in (5). For simplicity, the time argument is omitted when there is no possible confusion, meaning, more especially, that in the sequel $h, \zeta$ stand for $h(t)$ and $\zeta(t)$, respectively. Differentiating $V_{1}\left(x_{t}\right)$ along the trajectories of system (1) yields $\frac{\mathrm{d}}{\mathrm{d} t} V_{1}\left(x_{t}\right)=2 \tilde{x}^{T}(t) P \dot{\tilde{x}}(t)$. We then need to express $\tilde{x}(t)$ and $\dot{\tilde{x}}(t)$ using the augmented vector $\zeta$. On the one hand, it is easy to see that $\dot{x}(t)=\Sigma \zeta$. Moreover, simple calculations show that

$$
\begin{aligned}
& h_{1} \dot{\eta}_{1}(t)=\left[\begin{array}{ll}
e_{1}^{T}-e_{2}^{T} & e_{1}^{T}+e_{2}^{T}-2 e_{5}^{T}
\end{array}\right]^{T} \zeta \\
& \dot{\eta}_{6}(t)=\left[\begin{array}{ll}
e_{2}^{T}-e_{4}^{T} & \hat{G}_{0}^{T}
\end{array}\right]^{T} \zeta
\end{aligned}
$$

leading to $\dot{\tilde{x}}(t)=G_{0} \zeta$, where $G_{0}$ is defined in (7).

On the other hand, finding an expression $\tilde{x}(t)$ depending on $\zeta$ is more dedicated. We first note that $x(t)=e_{1} \zeta$ and $h_{1} \eta_{1}(t)=h_{1}\left[\begin{array}{cc}e_{5}^{T} & e_{6}^{T}\end{array}\right]^{T} \zeta$. Let us consider the last component of $\tilde{x}(t)$, i.e., $\eta_{6}(t)$. To express it using the extended state $\zeta$, we first note that

$$
\eta_{6}(t)=\left[\begin{array}{c}
\int_{-h}^{-h_{1}} x_{t}(s) \mathrm{d} s \\
h_{12} \int_{-h}^{-h_{1}} \delta_{4}(s) x_{t}(s) \mathrm{d} s
\end{array}\right]+\left[\begin{array}{c}
\int_{-h_{2}}^{-h} x_{t}(s) \mathrm{d} s \\
h_{12} \int_{-h_{2}}^{-h} \delta_{4}(s) x_{t}(s) \mathrm{d} s
\end{array}\right] .
$$

From this expression, we first note that the first $n$ components can be expressed as $\left(e_{11}+e_{13}\right) \zeta$. For the last $n$ components, we need to find two expressions of $\delta_{4}(s)$, which depend on $\delta_{2}(s)$ and $\delta_{3}(s)$, respectively. Some calculations show

$$
\begin{aligned}
& h_{12} \delta_{4}(s)=\left(h-h_{1}\right) \delta_{2}(s)+\left(h_{2}-h\right), \\
& h_{12} \delta_{4}(s)=\left(h_{2}-h\right) \delta_{3}(s)-\left(h-h_{1}\right) .
\end{aligned}
$$

Reinjecting (12) into (11) leads to

$$
\begin{aligned}
h_{12} & \left(\int_{-h}^{-h_{1}} \delta_{4}(s) x_{t}(s) \mathrm{d} s+\int_{-h_{2}}^{-h} \delta_{4}(s) x_{t}(s) \mathrm{d} s\right) \\
= & \left(h-h_{1}\right)\left(\int_{-h}^{-h_{1}} \delta_{2}(s) x_{t}(s) \mathrm{d} s-\int_{-h_{2}}^{-h} x_{t}(s) \mathrm{d} s\right) \\
& \quad+\left(h_{2}-h\right)\left(\int_{-h}^{-h_{1}} x_{t}(s) \mathrm{d} s+\int_{-h_{2}}^{-h} \delta_{3}(s) x_{t}(s) \mathrm{d} s\right) \\
= & \hat{G}_{1}(h) \zeta .
\end{aligned}
$$

Hence, we obtain that $\eta_{6}(t)=\left[e_{11}^{T}+e_{13}^{T} \hat{G}_{1}^{T}(h)\right] \zeta$, and $\tilde{x}(t)=G_{1}(h) \zeta$.

Moreover, from the definition of the augmented vector $\zeta$, one can see that the last four components can be seen as linear combination of the other components of $\zeta$, since we have $\eta_{4}(t)=\left(h-h_{1}\right) \eta_{2}(t)$ and $\eta_{5}(t)=\left(h_{2}-h\right) \eta_{3}(t)$. Therefore, using the matrices $g_{1}$ and $g_{2}$ defined in (8), the following equality holds for any matrices $N_{1}, N_{2}$ in $\mathbb{R}^{14 n \times 2 n}$

$$
2 \zeta^{T}\left(N_{1} g_{1}(h)+N_{2} g_{2}(h)\right) \zeta=0 .
$$

Therefore, the derivative of $V_{1}\left(x_{t}\right)$ is given by

$$
\frac{\mathrm{d}}{\mathrm{d} t} V_{1}\left(x_{t}\right)=\zeta^{T} \operatorname{He}\left(G_{1}^{T}(h) P G_{0}+N_{1} g_{1}(h)+N_{2} g_{2}(h)\right) \zeta
$$

The differentiation of $V_{2}\left(x_{t}\right)$ along the trajectories of (1) leads to $\frac{\mathrm{d}}{\mathrm{d} t} V_{2}\left(x_{t}\right)=\zeta^{T} \hat{S} \zeta$ and using Lemmas 1 and 2 as in [5], the derivative of $V_{3}\left(x_{t}, \dot{x}_{t}\right)$ can be upper bounded by $\zeta^{T}\left(\Sigma^{T}\left(h_{1}^{2} R_{1}+h_{12}^{2} R_{2}\right) \Sigma-G_{2}^{T} \tilde{R}_{1} G_{2}-\Gamma^{T} \Psi \Gamma\right) \zeta$. Finally, combining the previous expressions, we have

$$
\frac{\mathrm{d}}{\mathrm{d} t} V\left(x_{t}, \dot{x}_{t}\right) \leq \zeta^{T} \Phi(h) \zeta
$$

where $\Phi(h)$ is given in (7). Since $\Phi(h)$ is affine, and consequently convex, with respect to $h$, the two LMIs $\Phi\left(h_{1}\right) \prec 0$ and $\Phi\left(h_{2}\right) \prec 0$ imply $\Phi(h) \prec 0$ for all $h \in\left[h_{1}, h_{2}\right]$. Therefore, LMIs (6) imply the asymptotic stability of system (1) for any delay verifying (2).

\subsection{A second stability result}

The conditions of Theorem 1 are derived by virtue of Lemmas 1 and 2. Alternatively, a similar analysis can be performed by substituting Lemma 2 for Lemma 3, leading to the next theorem.

Theorem 2 If there exist matrices $P \in \mathbb{S}_{+}^{5 n}, S_{1}, S_{2}$, $R_{1}, R_{2} \in \mathbb{S}_{+}^{n}$, and matrices $N_{1}, N_{2} \in \mathbb{R}^{14 n \times 2 n}$, and two matrices $Y_{1}, Y_{2} \in \mathbb{R}^{14 n \times 3 n}$, such that the LMIs

$$
\left[\begin{array}{cc}
\Phi_{0}\left(h_{i}\right)-\operatorname{He}\left(Y_{2} G_{3}+Y_{1} G_{4}\right) & Y_{i} \\
Y_{i}^{T} & -\tilde{R}_{2}
\end{array}\right] \prec 0,
$$

hold for $i=1,2$, where matrices $G_{3}, G_{4}$ and $\Phi_{0}, \tilde{R}_{2}$ are given in (4) and (7), then system (1) is asymptotically stable for any delay function $h$ satisfying (2).

Proof: The proof follows the same lines of reasoning as the proof of Theorem 1. The main modification is the application of Lemma 2 replaced by Lemma 3, which has been widely used in the literature and is therefore omitted.

\subsection{Comparison of Theorems 1 and 2}

In this section, we provide a comparison of the conservatism of the two previous theorems. The main idea is to demonstrate that if the conditions of Theorem 1 are verified, then there also exists a solution to the conditions 
Table 1

\begin{tabular}{|c|c|c|c|c|c|c|c||c|}
\hline$h_{1}$ & 0.0 & 0.4 & 0.7 & 1.0 & 2.0 & 3.0 & $h_{1}=h_{2}$ & No. of variables \\
\hline \hline$[4]$ & 1.86 & 1.88 & 1.95 & 2.06 & 2.61 & 3.31 & 4.47 & $3.5 n^{2}+2.5 n$ \\
{$[9]$} & 2.11 & 2.17 & 2.23 & 2.31 & 2.79 & 3.49 & 6.05 & $10.5 n^{2}+3.5 n$ \\
{$[5]$} & 2.14 & 2.19 & 2.24 & 2.31 & 2.80 & 3.50 & 6.1641 & $21 n^{2}+6 n$ \\
{$[11]$} & 2.18 & 2.21 & 2.25 & 2.32 & 2.79 & 3.49 & 6.05 & $54.5 n^{2}+9.5 n$ \\
\hline \hline Theorem 1 & 2.20 & 2.26 & 2.32 & 2.38 & 2.81 & 3.50 & 6.1664 & $79.5 n^{2}+4.5 n$ \\
\hline Theorem 2 & 2.26 & 2.29 & 2.34 & 2.40 & 2.83 & 3.50 & 6.1664 & $154.5 n^{2}+4.5 n$ \\
\hline
\end{tabular}

Example 1: admissible upper bound of $h_{2}$ for different $h_{1}$

of Theorem 2. Indeed, assuming that the conditions of Theorem 1 and selecting, in Theorem 2,

$$
Y_{1}^{T}=\tilde{R}_{2} G_{4}+X^{T} G_{3}, Y_{2}^{T}=\tilde{R}_{2} G_{3}+X G_{4}
$$

we obtain

$$
\begin{aligned}
& \Phi_{0}\left(h_{1}\right)-\operatorname{He}\left(Y_{2} G_{3}+Y_{1} G_{4}\right)+Y_{1} \tilde{R}_{2}^{-1} Y_{1}^{T} \\
& \quad=\Phi_{0}\left(h_{1}\right)-\Gamma^{T}\left(\Psi+\left[\begin{array}{cc}
\tilde{R}_{2}-X \tilde{R}_{2}^{-1} X^{T} & 0 \\
0 & 0
\end{array}\right]\right) \Gamma \\
& \quad \leq \Phi_{0}\left(h_{1}\right)-\Gamma^{T} \Psi \Gamma
\end{aligned}
$$

where $\Gamma$ and $\Psi$ are given in (4) and (6), respectively. The latter inequality is guaranteed by application of the Schur complement to (6). A similar analysis yields the same result when $h(t)=h_{2}$, which allows concluding the comparison. Hence, the comparison reveals that Theorem 2 always produces better (or at least the same) results than Theorem 1. As usual, the potential improvement of Theorem 2 over Theorem 1 is at the price of a notable increase of the number of decision variables, showing again a tradeoff between the reduction of the conservatism and the numerical complexity.

\section{Illustrative Examples}

Three numerical examples from the literature will illustrate the efficiency of the proposed conditions.

\subsection{Example 1}

Consider the following much-studied system (1) with

$$
A=\left[\begin{array}{rr}
-2.0 & 0.0 \\
0.0 & -0.9
\end{array}\right], A_{1}=\left[\begin{array}{rr}
-1.0 & 0.0 \\
-1.0 & -1.0
\end{array}\right] \text {, }
$$

which is well-known for being asymptotically stable for all constant delays lower than $h_{\max }=6.1725$. Table 1 shows that the maximum allowable delays $h_{2}$ for several

\begin{tabular}{|c|c|c|c|c|c|}
\hline$h_{1}$ & 0.0 & 0.3 & 0.7 & 1.0 & 2.0 \\
\hline \hline$[9]$ & 1.59 & 2.01 & 2.41 & 2.62 & 3.59 \\
{$[5]$} & 1.64 & 2.13 & 2.70 & 2.96 & 3.63 \\
{$[11]$} & 1.80 & 2.19 & 2.58 & 2.79 & 3.68 \\
\hline \hline Theorem 1 & 2.04 & 2.47 & 2.87 & 3.11 & 3.77 \\
\hline Theorem 2 & 2.39 & 2.76 & 3.15 & 3.41 & 4.04 \\
\hline
\end{tabular}

Table 2

Example 2: admissible upper bound of $h_{2}$ for different $h_{1}$

values of $h_{1}$ obtained by Theorems 1 and 2 are less conservative than those obtained by various recent methods from the literature. Moreover, compared to Theorem 1, Theorem 2 slightly improves the results at the price of $75 n^{2}$ additional decision variables. Furthermore, by applying Theorems 1 and 2, we find that for any constant delay in the interval $[0,6.1664]$ the system remains asymptotically stable, which is consistent with the analysis provided in $[8,12]$, which are based on the same integral inequality.

\subsection{Example 2}

Consider system (1) from [5] with

$$
A=\left[\begin{array}{rr}
0.0 & 1.0 \\
-10.0 & -1.0
\end{array}\right], \quad A_{1}=\left[\begin{array}{ll}
0.0 & 0.1 \\
0.1 & 0.2
\end{array}\right]
$$

Table 2 presents admissible upper bound of $h_{2}$ for different $h_{1}$. We can see that Theorem 1 essentially improves the results. Moreover, in this example, Theorem 2 reduces notably the conservatism compared with Theorem 1, of course at the price of an increase of the number of decision variables (see the last column of Table 1).

\section{$4.3 \quad$ Example 3}

Theorems 1 and 2 can be also applied to the stability analysis of system (1) with interval delays, which may be unstable for small delays (or without delays). Consider 


\begin{tabular}{|c|c|c|c|c|c|c|}
\hline$h_{1}$ & 0.11 & 0.3 & 0.5 & 0.8 & 1.0 & 1.3 \\
\hline$[11]$ & 0.40 & 1.09 & 1.34 & 1.49 & 1.53 & 1.54 \\
{$[9]$} & 0.42 & 1.09 & 1.36 & 1.52 & 1.56 & 1.57 \\
{$[5]$} & 0.40 & 1.11 & 1.40 & 1.59 & 1.65 & 1.70 \\
\hline \hline Theorem 1 & 0.50 & 1.23 & 1.46 & 1.63 & 1.68 & 1.70 \\
\hline Theorem 2 & 0.50 & 1.23 & 1.46 & 1.63 & 1.69 & 1.70 \\
\hline
\end{tabular}

Table 3

Example 3: admissible upper bound of $h_{2}$ for different $h_{1}$

system (1) with

$$
A=\left[\begin{array}{rr}
0.0 & 1.0 \\
-2.0 & 0.1
\end{array}\right], \quad A_{1}=\left[\begin{array}{ll}
0.0 & 0.0 \\
1.0 & 0.0
\end{array}\right]
$$

The frequency domain approach for constant delays shows that this system is stable if the delay belongs to the interval $[0.100169,1.7178]$ [2]. Table 3 shows again that Theorems 1 and 2 are much less conservative than the ones from the literature.

For the constant delay case, where $h_{1}=h_{2}$, it was found in [9] that the system remains stable for all constant delays in the interval $[0.100529,1.5405]$. The conditions of [11] lead to an interval [0.100509, 1.5404]. The result obtained by solving the conditions from Theorems 1 or 2 with $h_{1}=h_{2}$ leads to stability of this system for all constant delays in the interval [0.100169, 1.7122], which is also consistent with the results of [12] and very close to the analytical one.

\section{Conclusions}

Two novel stability criteria of systems with time-varying delays have been presented in this paper. The construction of augmented LKFs and the derivation of the novel stability criteria have fully considered the new features of the second-order Bessel-Legendre integral inequality. Further research aims at reducing the computational complexity. Indeed we have shown that the reduction of the conservatism is related to both the refinement of the integral inequality and the state augmentation in the LKF. However, the state augmentation leads, in the time-varying delay case, to conditions that are polynomial in $h$, and that cannot be easily treated, constituting a direction for future research.

\section{References}

[1] E. Fridman. Introduction to Time-Delay Systems. Birkhäuser, 2014.

[2] K. Gu, V. Kharitonov, and J. Chen. Stability of Time-Delay Systems. Birkhäuser, Boston, 2003.

[3] Y.S. Moon, P.G. Park, W.H. Kwon, and Y.S. Lee. Delaydependent robust stabilization of uncertain state-delayed systems. International Journal of Control, 74(14):1447-1455, 2001.
[4] P.G. Park, J.W. Ko, and C. Jeong. Reciprocally convex approach to stability of systems with time-varying delays. Automatica, 47(1):235-238, 2011.

[5] P.G. Park, W.I. Lee, and S.Y. Lee. Auxiliary functionbased integral inequalities for quadratic functions and their applications to time-delay systems. Journal of the Franklin Institute, 352(4):1378-1396, 2015.

[6] A. Seuret and F. Gouaisbaut. Wirtinger-based integral inequality: application to time-delay systems. Automatica, 49(9):2860-2866, 2013.

[7] A. Seuret and F. Gouaisbaut. Complete quadratic Lyapunov functionals using Bessel-Legendre inequality. In Proceedings of 2014 ECC, Strasbourg, France, June 2014.

[8] A. Seuret and F. Gouaisbaut. Hierarchy of LMI conditions for the stability analysis of time-delay systems. Systems $\mathbb{E}$ Control Letters, 81:1-7, 2015.

[9] A. Seuret, F. Gouaisbaut, and E. Fridman. Stability of systems with fast-varying delay using improved wirtinger's inequality. In Proceedings of the 52nd IEEE CDC, Florence, Italy, December 2013.

[10] S. Y. Xu, J. Lam, B. Y. Zhang, and Y. Zou. New insight into delay-dependent stability of time-delay systems. International Journal of Robust and Nonlinear Control, 25(7):961-970, 2015.

[11] H.B. Zeng, Y. He, M. Wu, and J.H. She. Free-matrix-based integral inequality for stability analysis of systems with timevarying delay. IEEE Transactions on Automatic control, 60(10):2768-2772, 2015.

[12] H.B. Zeng, Y. He, M. Wu, and J.H. She. New results on stability analysis for systems with discrete distributed delay. Automatica, 60:189-192, 2015. 